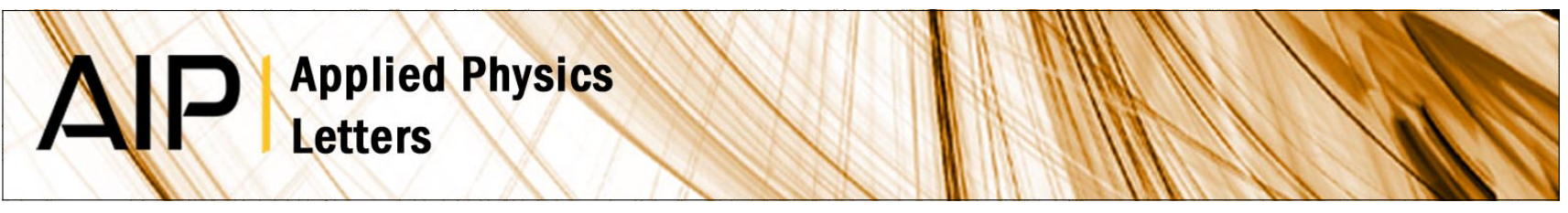

\title{
Photoacoustic determination of thermal diffusivity of solids: Application to CdS
}

C. L. Cesar, H. Vargas, J. Mendes Filho, and L. C. M. Miranda

Citation: Appl. Phys. Lett. 43, 555 (1983); doi: 10.1063/1.94417

View online: http://dx.doi.org/10.1063/1.94417

View Table of Contents: http://apl.aip.org/resource/1/APPLAB/v43/i6

Published by the American Institute of Physics.

Additional information on Appl. Phys. Lett.

Journal Homepage: http://apl.aip.org/

Journal Information: http://apl.aip.org/about/about_the_journal

Top downloads: http://apl.aip.org/features/most_downloaded

Information for Authors: http://apl.aip.org/authors

\section{ADVERTISEMENT}

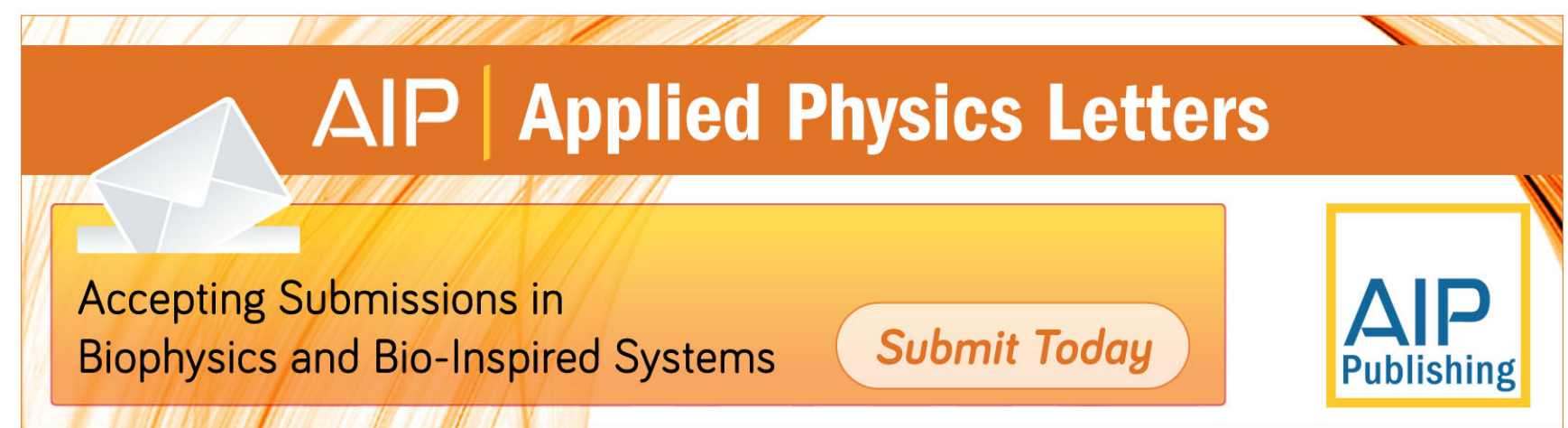




\title{
Photoacoustic determination of thermal diffusivity of solids: Application to CdS
}

\author{
C. L. Cesar, H. Vargas, and J. Mendes Filho \\ Instituto de Física, Universidade Estadual de Campinas, 13100. Campinas. SP. Brazil \\ L. C. M. Miranda \\ Instituto de Estudos Avançados, Centro Técnico Aeroespacial, 12200, S. J. Campos, SP. Brazil
}

(Received 29 March 1983; accepted for publication 11 July 1983)

A novel and simple approach, which uses a lateral heating source for the sample, is proposed as an alternative method for the photoacoustic determination of the thermal diffusivity of solids. The method is experimentally tested using a CdS sample.

PACS numbers: $66.70 .+\mathrm{f}, 65.90 .+\mathrm{i}$

It has been well recognized ${ }^{1-8}$ that photoacoustic spectroscopy (PAS) provides a sensitive means for measuring the optical $^{2.3}$ and thermal properties ${ }^{4-7}$ of solids. According to the existing approaches, the determination of these properties, however, involves the normalization of two signals. ${ }^{2-4,6}$ In particular, for determining the thermal diffusivity, one method ${ }^{4}$ consists in measuring the signal $\left(S_{2}\right)$ obtained when the light beam is incident from the sample side, and that obtained when it is incident on the window side $\left(S_{1}\right)$. For the case of a highly absorbing sample the ratio $S_{2} / S_{1}$ is given by ${ }^{4}$

$$
S_{2} / S_{1}=\left[\cosh ^{2}\left(\pi f^{2} / \alpha\right)^{1 / 2}-\sin ^{2}\left(\pi f^{2} / \alpha\right)^{1 / 2}\right]^{-1 / 2},(1)
$$

where $\alpha$ is the thermal diffusivity, $f$ is the modulation frequency, and $l$ is the sample length. A least-square fit of the data, as a function of the modulation frequency, to Eq. (1) allows us the determination of the thermal diffusivity. The disadvantage of this approach is that one has to measure two signals, on different sides of the sample, at the same surface conditions. It is not usually possible to overcome these difficulties so that additional errors, which may affect the accuracy of our results, are introduced in the normalization process.

In the following we describe an alternative single-signal approach for determining the thermal diffusivity of optically transparent samples. This novel approach is schematically shown in Fig. 1. It basically uses the optical transparency of the sample to a given wavelength for generating, through a lateral modulated illumination, a periodic heat source at a point $\chi_{0}$ of the sample of the form

$$
S(x, t)=\beta^{\prime} I_{0} \delta\left(x-x_{0}\right) e^{j w o t} ; \quad \beta^{\prime}=\beta d .
$$

Here $I\left(\mathrm{~W} / \mathrm{cm}^{2}\right)$ is the incident monochromatic light intensity, $\beta\left(\mathrm{cm}^{-1}\right)$ is the bulk optical absorption coefficient at the light wavelength, $\omega=2 \pi f$ is the modulation frequency, and $d(\mathrm{~cm})$ is the lateral thickness of the sample. Equation (2) represents, to a good approximation, the thermal power density generated at a point $x=x_{0}$ when a laser beam crosses the sample of thickness $d$. Using Eq. (2) in the Rosencwaig and Gersho' (RG) one-dimensional model for the heat flow in the PA cell resulting from the absorption of modulated light, the thermal diffusion equation in the sample is written as $1,2.8$

$$
\frac{\partial^{2} \phi_{s}}{\partial x^{2}}-\frac{1}{\alpha} \frac{\partial \phi_{s}}{\partial t}=-\frac{S(x, t)}{k_{s}}-l<x<0,
$$

where $\phi_{s}$ is the temperature fluctuation in the sample, $\alpha_{s}$ $=k_{s} / \rho_{s} c_{s}$ is the thermal diffusivity of the sample, $k_{s}$ is the thermal conductivity, $\rho_{s}$ is the material density, and $c_{s}$ is the specific heat of the sample. For the gas $(g)$ and backing $(b)$ materials, the thermal diffusion equations are given by

$$
\begin{aligned}
& \frac{\partial^{2} \phi_{g}}{\partial x^{2}}=\frac{1}{\alpha_{g}} \frac{\partial \phi_{g}}{\partial t} \quad 0<x<l g, \\
& \frac{\partial^{2} \phi_{b}}{\partial x^{2}}=\frac{1}{\alpha_{b}} \frac{\partial \phi_{b}}{\partial t} \quad-\left(l+l_{b}\right)<x<l .
\end{aligned}
$$

Equations (3) and (4) together with the boundary conditions requiring the temperature and heat-flux continuity at the boundaries $x=0$ and $x=-l$, and that the cell walls are at ambient temperature $T_{0}$. [i.e., $\phi_{g}\left(l_{g}\right)=\phi_{b}\left(-l-l_{b}\right)=0$ ] completely specify the temperature distribution in the cell. It should be emphasized that this one-dimensional model is justifiable provided the sample is transparent at the operating wavelength.

Neglecting transients as well as the de components, and assuming that the gas and the backing are thermally thick (i.e., thermal diffusion length small compared to the material length), the temperature fluctuation in the gas is found to be

$$
\phi_{g}(x, t)=\theta e^{j \omega t},
$$

where

$$
\begin{aligned}
& \theta=\frac{\beta^{\prime} I_{0}}{k_{s} \sigma_{s}} \\
& \times \frac{\left[(1-b) e^{k}+(1+b) e^{+\omega_{1}}\right]}{\left[(1-b)(1-g) e^{\omega_{N}}-(1+b)(1+g) e^{+\omega_{1}}\right]} \\
& \times e^{\sigma_{1}, x_{1}} \text {. }
\end{aligned}
$$

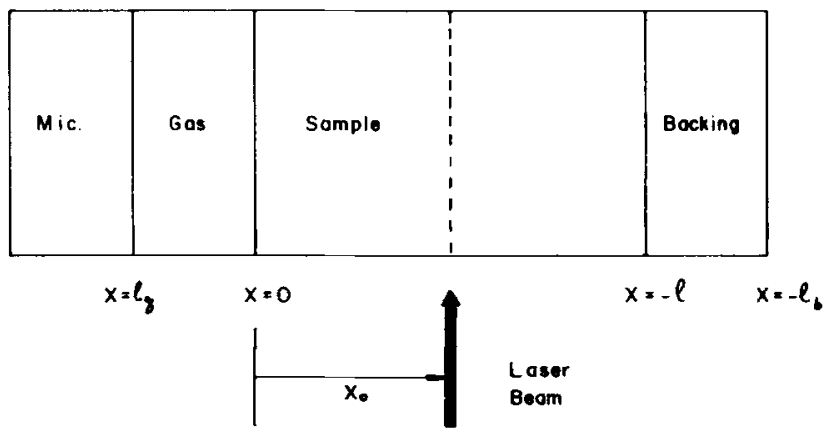

FIG. 1. Schematic configurations of the photoacoustic cell for determining the thermal diffusivity. 


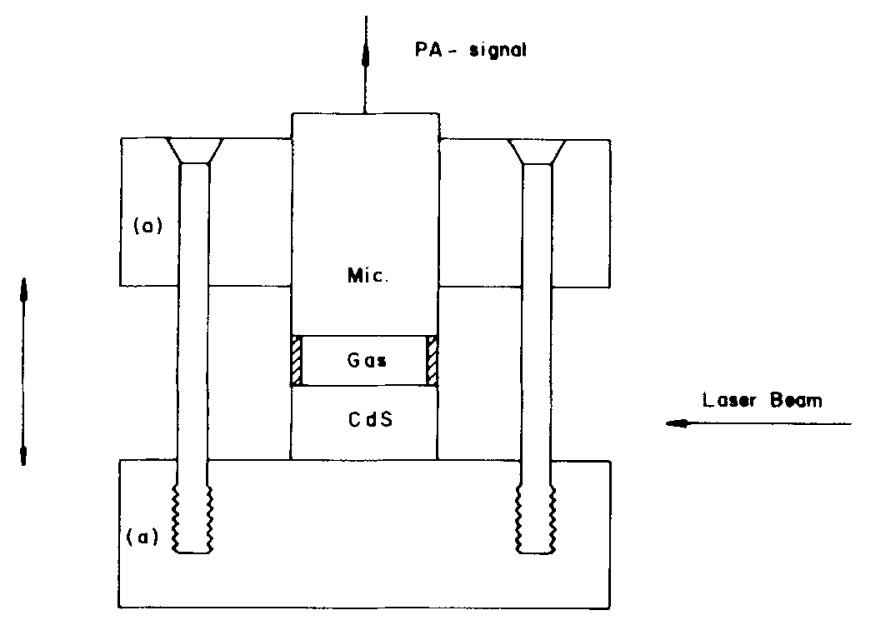

FIG. 2. Experimental setup used for determining the thermal diffusivity of $\mathrm{CdS}$, in which the brass discs (a) are connected by two screws. The arrow on the left-hand side indicates that the whole system could move up and down by means of a micrometer positioner mounted on the lower disc.

Here, $g=k_{g} \sigma_{g} / k_{s} \sigma_{s}, b=k_{b} \sigma_{b} / k_{s} \sigma_{s}, \sigma_{s}=(1+j) a_{y}$, and $a_{s}$ $=\left(\pi f / \alpha_{s}\right)^{i / 2}$ is the thermal diffusion coefficient $\left(\mathrm{cm}^{-1}\right)$ of the sample.

Once we know the temperature fluctuation in the gas, given by Eqs. (5), we can then assume the $R G$ acoustic-piston model' for evaluating the pressure fluctuations $\delta P(t)$ in the gas. One gets

$$
P(t)=\frac{\gamma P_{0} \theta}{T_{0} l_{g} \sigma_{g}} \epsilon^{j \omega t},
$$

where $P_{0}$ is the ambient pressure, $\gamma$ is the ratio of the specific heats of the gas, and $\sigma_{g}=(1+j) a_{g}$. Equation (6) is the expression for the PA signal one wants to discuss. We note from Eqs. (5) and (6) that, in the present experimental configuration, the PA signal exhibits an exponential dependence on the product $a_{s} x_{0}$ between the illuminated region position $x_{0}$ and the thermal diffusion coefficient $a_{s}=\left(\pi f / \alpha_{s}\right)^{1 / 2}$. This means that by measuring the acoustic signal, at a fixed modulation frequency, as a function of $x_{0}$ the thermal diffusivity is readily obtained, as the coefficient of $x_{0}$ in the exponential.

To test the above method for determining $\alpha_{s}$ we mea-

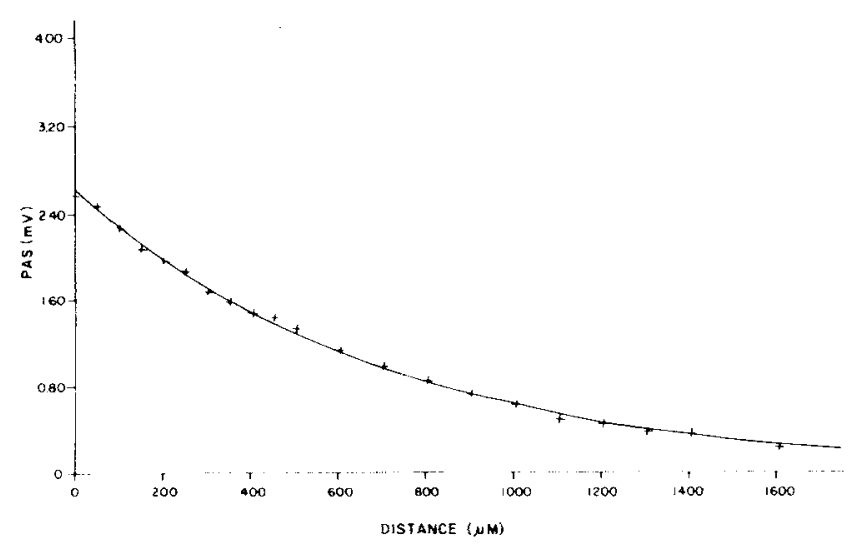

FIG. 3. Amplitude of the PA signal of a CdS sample as a function of the illuminated region position $x_{i}$, using an argon laser light modulated at 10.25 $\mathrm{Hz}$. The crosses refer to the experimental results, whereas the solid curve is the fitting of the data to an expression of the form $S=S_{0} \exp \left(-a_{1}, x_{0}\right)$.

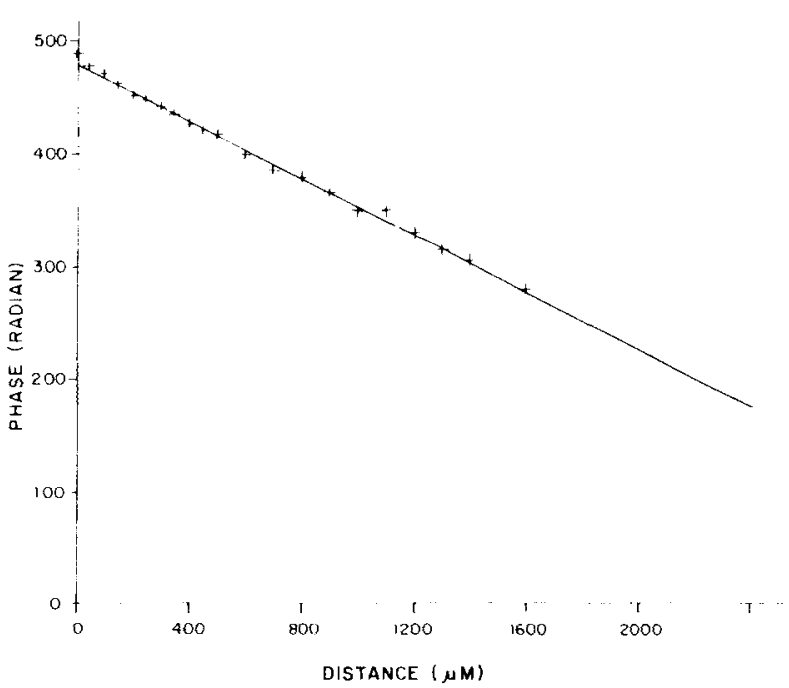

FIG. 4. Phase variation of the PA signal of a CdS sample as a function of the illuminated region position $x_{0}$, using an argon laser light modulated at 10.25 Hz. The crosses refer to the experimental results, whereas the solid curve is the fitting of the data to an expression $\psi-\psi_{0}=-a, x_{11}$

sured the PA signal of $\operatorname{CdS}^{9}\left(l_{s}=9 \mathrm{~mm}, d=8.2 \mathrm{~mm}, C_{s}\right.$ $=0.37 \mathrm{~J} / \mathrm{g}{ }^{\circ} \mathrm{C}, \rho_{s}=4.82 \mathrm{~g} / \mathrm{cm}^{3}, k_{s}=0.27 \mathrm{~W} / \mathrm{cm}^{\circ} \mathrm{C}$ ) as a function of $x_{0}$. The experiment was done using the light from an argon laser (Coherent Radiation-CR8) modulated at a frequency of $f=10.25 \mathrm{~Hz}$. This ensured us the condition of optical quasitransparency of the sample (i.e., $\beta d<1$ ). The PA cell is schematically shown in Fig. 2, and consists basically of two brass discs on which the sample and the microphone were mounted. The microphone used was a BK4166 capable of providing a flat frequency response of $50 \mathrm{mV} / \mathrm{Pa}$ from $3.0 \mathrm{~Hz}$ to $10 \mathrm{kHz}$. The laser beam had a beam waist of roughly $0.1 \mathrm{~mm}$ and its power was constantly monitored in each measurement. The whole PA setup could be moved up and down by means of micrometer positioner attached to one of the brass discs. In this way we could vary the illumination region position $x_{0}$. In Figs. 3 and 4 we plot the observed amplitude and phase of the acoustic signal as a function of $x_{0}$, respectively. Here we note that, because of the finite waist of the laser beam, the illumination position is defined relative to the beam waist. That is, suppose that we are illuminating the sample from the gas side. As soon as part of the beam reaches the sample, the PA signal begins to grow; on further moving the sample, the PA signal eventually saturates when the entire beam is illuminating the sample. Up to this point we have moved the sample a distance of roughly $100 \mu \mathrm{m}$, which corresponds to the beam waist. From this point on, the PA signal decreases when moving the sample. Thus, when plotting our data we have subtracted the beam waist in the illumination position scale. Fitting the experimental data to an expression of the form [cf., Eqs. (5) and (6)]

$$
S=S_{0} e^{-\sigma_{0} x_{0}}=|S| e^{i \psi^{\prime \prime}},
$$

where

$$
\begin{aligned}
& |S|=S_{0} e^{a_{v} x_{0}} \\
& \psi=\psi_{0}-a_{s} x_{0},
\end{aligned}
$$

we have determined the coefficient $a_{s}=\left(\pi f / \alpha_{s}\right)^{1 / 2}$, from which the thermal diffusivity was deduced to be $\alpha_{4}=0.16$ 
$\mathrm{cm}^{2} / \mathrm{s}$. This value of $\alpha_{s}$ should then be compared with the known value of thermal diffusivity of $\mathrm{CdS},{ }^{4}$ namely, $\alpha=0.15 \mathrm{~cm}^{2} / \mathrm{s}$. In our view, this extremely good agreement between the calculated value of $\alpha$ and the known value of it, together with the simplicity of the experimental setup, strongly supports the present photoacoustic method for determining the thermal diffusivity of solids.

'A. Rosencwaig and A. Gersho. J. Appl. Phys. 47, 64 (1976).

'G. C. Wetsel, Jr. and F. A. MacDonald. Appl. Phys. Lett. 30. 252 (1977).
${ }^{3}$ A. C. Tam, C. K. N. Patel, and R. J. Kerl, Opt. Lett. 4. 81।1979|.

${ }^{\dagger} Z$. Yasa and N. M. Amer. Topical Meeting on Photoacoustic Spectroscopy, Ames 1979. paper WA5-1.

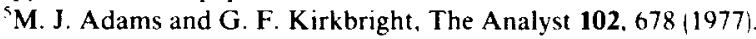

"F. Lepoutre. P. Charpentier. C. Boccara, and D. Fournier. Topical Meeting on Photoacoustic Spectroscopy. Berkeley 1981. paper MA3-1.

${ }^{7}$ C. C. Ghizoni, M. A. A. Siqueira. H. Vargas, and L. C. M. Miranda, Appl. Phys. Lett. 32. 554 (1978).

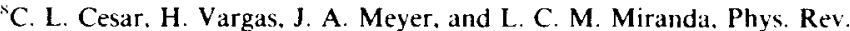
Lett. 42. $1570(1979)$.

". Properties of the II-VI crystals," Information Sheet. Cleveland Crystals Inc., August 1978. 